\title{
A case report of unilateral conductive hearing loss as a consequence of malignant sinonasal melanoma: the importance of completing a full clinical history and examination
}

\author{
Victoria Blackabey * (D, Olivia Kenyon and Rishi Talwar
}

\begin{abstract}
Background: Sinonasal melanoma is a rare head and neck tumour. It is associated with a poor prognosis, high rates of loco-regional recurrence and distant metastasis. Treatment of the disease is therefore complicated, and because of limited data regarding the cancer, management is frequently tailored to the individual patient. We describe an unusual presentation of sinonasal melanoma with relevant histology, radiology and clinical photography.

Case presentation: The case report describes the presentation of a 64-year-old man to the Ear, Nose and Throat department with progressive right-sided hearing loss. A thorough history highlighted other clinical symptoms including unilateral nasal obstruction and epistaxis. Clinical examination showed a right middle ear effusion with a polypoidal lesion in the right nasal cavity. Relevant imaging demonstrated a destructive process that required further assessment. An endoscopic sinus procedure was performed to obtain histological diagnosis as well as providing symptomatic relief. Histology confirmed malignant mucosal melanoma. The patient underwent maxillectomy and orbital exenteration (due to further progression of disease) at a tertiary centre with a plan for subsequent immunotherapy. This however has been delayed due to further surgery to excise a metastatic lesion to the right femur.

Conclusions: This case report highlights the importance of a thorough clinical history and examination. An unusual presentation of a sinonasal tumour can easily be missed leading to a significant delay in treatment. The case report also describes the use of functional endoscopic sinus surgery in order to obtain histological diagnosis and to debulk the tumour, providing symptomatic relief. The current literature regarding management will be discussed as well as current developments guiding future treatment.
\end{abstract}

Keywords: Sinonasal melanoma, Case report, Maxillary sinus, Otitis media with effusion, Conductive hearing loss

\footnotetext{
* Correspondence: v.blackabey@nhs.net

Department of Otolaryngology, Luton and Dunstable University Hospital, Lewsey road, Luton LU4 ODZ, UK
}

\section{Springer Open}

(c) The Author(s). 2020 Open Access This article is licensed under a Creative Commons Attribution 4.0 International License, which permits use, sharing, adaptation, distribution and reproduction in any medium or format, as long as you give appropriate credit to the original author(s) and the source, provide a link to the Creative Commons licence, and indicate if changes were made. The images or other third party material in this article are included in the article's Creative Commons licence, unless indicated otherwise in a credit line to the material. If material is not included in the article's Creative Commons licence and your intended use is not permitted by statutory regulation or exceeds the permitted use, you will need to obtain permission directly from the copyright holder. To view a copy of this licence, visit http://creativecommons.org/licenses/by/4.0/. 


\section{Background}

Mucosal melanoma of the sinonasal cavity accounts for $5 \%$ of head and neck mucosal melanomas that is estimated to be $25-30 \%$ of total melanomas [1]. Like other melanomas, it is associated with a poor prognosis, high rates of locoregional recurrence and distant metastasis, often as a result of late presentation $[1,2]$. The 5-year survival rate ranges between 15 and $28 \%$, with median survival less than 2 years following diagnosis [2].

Melanocytes arise embryologically from the neuroectoderm and are found in the basal layer of the skin and some mucosal surfaces, such as the mucosa of the sinonasal cavity [3]. Unlike cutaneous melanoma, in which UV light exposure is a known risk factor, there is no evidence of associated risk factors in mucosal melanoma [2, 3]. However, research has hypothesised that air pollution and occupational exposure to formaldehyde may be contributing factors [4-6].

Sinonasal mucosal melanoma usually presents in patients between 65 and 70 years of age [6]. It is more prevalent in both Caucasian and Asian populations [6]. Although there is no difference in gender prevalence, the incidence is currently increasing in women $[3,6]$.

The most common presenting symptoms include epistaxis and nasal obstruction [6]. Other symptoms include epiphora, anosmia and rhinorrhoea $[3,6]$. This is likely due to tumour location and progression, which often arises from the nasal septum, inferior and middle turbinates and lateral nasal wall $[3,6,7]$. Only $20 \%$ of tumours arise from the sinuses $[3,6,7]$. At present, a third of patients show evidence of neck or distant metastases [7].

Worldwide published guidelines describe the mainstay of treatment for sinonasal melanoma to be radical surgery with the aim of clear surgical margins $[2,6]$. However, the main consequence of such radical treatment is severe functional loss. Therefore, it is not currently indicated where metastasis has been proven $[2,6]$. Primary and adjuvant radiotherapy and chemotherapy have been used with varying results $[2,6]$.

We describe an unusual presentation of sinonasal melanoma and discuss current literature regarding management and future therapeutic developments.

\section{Case presentation}

A 64-year-old man presented to the Ear, Nose and Throat clinic with a 4-month history of worsening right sided hearing loss. He also described progressive unilateral nasal obstruction and epistaxis. On direct questioning, he reported worsening hyposmia, facial paraesthesia in the region of right $V_{2}$ (maxillary branch of trigeminal nerve) as well as right epiphora.
On clinical examination, a polypoidal lesion was obstructing the right nasal cavity. Oral and neck examination was otherwise unremarkable. Otoscopy revealed findings consistent with a right middle ear effusion. Pure tone audiogram demonstrated a mild-to-moderate unilateral conductive hearing loss.

Computed tomography (CT) of the sinuses demonstrated a polypoidal lesion obstructing the right nasal cavity as well as opacification of the right maxillary sinus (Fig. 1). There was also obstruction of the frontal, anterior and posterior ethmoidal sinuses. Furthermore, the $\mathrm{CT}$ showed loss of the lamina papyracea with invasion of the lesion into the right orbit and displacement of the right inferior rectus muscle. Although the $\mathrm{CT}$ report suggested a possible benign aetiology, magnetic resonance imaging (MRI) was requested to further characterise the lesion.

MRI sinuses confirmed extension of the lesion from the right maxillary sinus into the right nasal cavity and ethmoid sinuses (Fig. 2). Invasion into the right orbit, displacing the right inferior rectus muscle was demonstrated. Additionally, there was opacification of the right mastoid air cells and evidence of a right middle ear effusion.

As part of the investigative process, he was tested for HIV (which was negative) and referred to ophthalmology for further assessment of his vision.

Differential diagnoses included inverted papilloma, squamous cell carcinoma (SCC), undifferentiated carcinoma, adenocarcinoma, rhabdomyosarcomas and lymphoma. Functional endoscopic sinus surgery (FESS) was

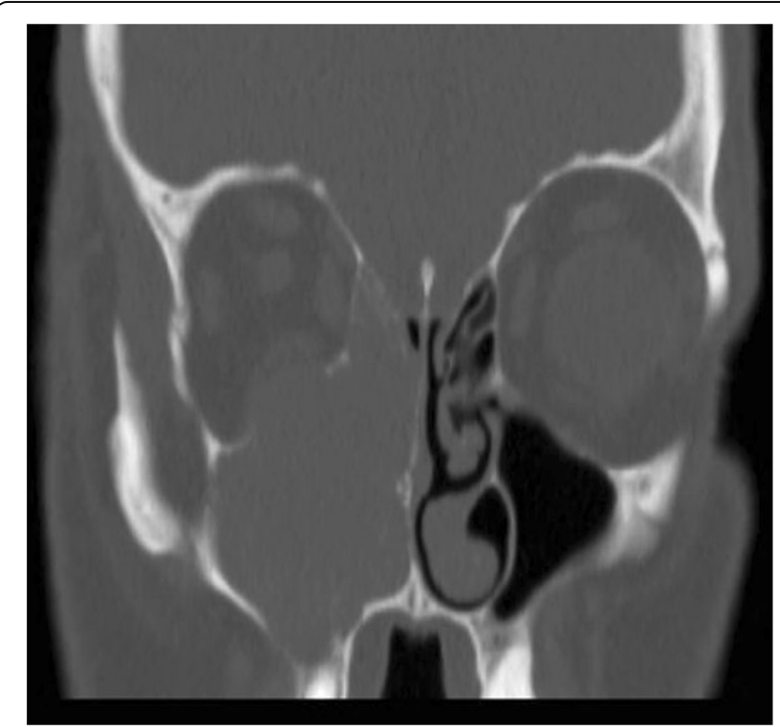

Fig. $1 \mathrm{CT}$ coronal view demonstrating a lesion within the right maxillary sinus and nasal cavity, eroding the right lamina papyracea as well as involvement of the right inferior rectus muscle 


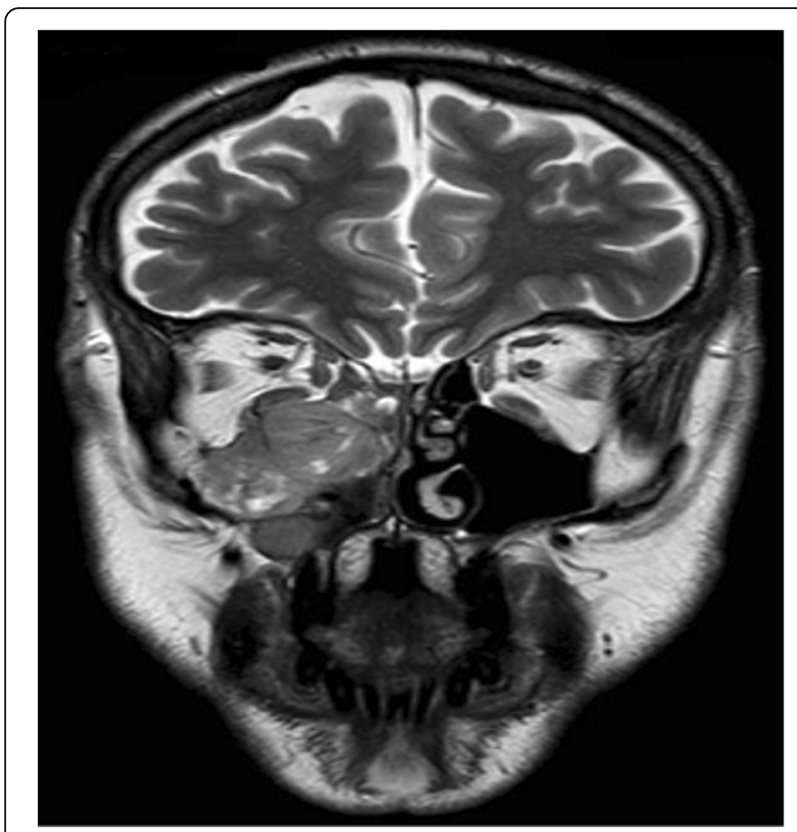

Fig. $2 \mathrm{MRI}$ coronal view demonstrating a heterogenous lesion involving right maxillary and ethmoid sinuses and confirming involvement of the right orbit

performed (Fig. 3) in which the lesion was debulked and biopsies were taken from multiple sites within the nasal cavity, postnasal space (PNS) and sinuses. It was decided not to insert a ventilation tube but to provide the patient with a hearing aid. This minimises the postoperative risk of a constantly discharging ear that may ultimately affect the use chemoradiotherapy. If enough of the tumour could be debulked, this may improve eustachian tube drainage and thereby allow the effusion to resolve.

Histology showed patchy necrosis composed of poorly differentiated and pleomorphic cells with a high mitotic index. Melanin was focally identified and, following immunohistochemistry, confirmed as malignant melanoma.

Following diagnosis, the patient was referred to a tertiary centre where he had a maxillectomy and orbital exenteration (due to progression of disease). Following this, the plan was to perform immunotherapy. However, this has been delayed due to a metastatic lesion in the right femur which was to be excised prior to proceeding with further management.

\section{Discussion}

Evidence-based management is difficult due to the rarity of sinonasal mucosal melanoma. The mainstay of treatment remains radical surgery with clear surgical margins $(1.5-2 \mathrm{~cm})$ [7]. Without clear margins, the risk of local and distant spread increases [8]. Extensive surgery however impacts both function and aesthetics, and therefore, the patient's co-morbidities and quality of life should be considered [6, 7].

FESS remains controversial due to difficulty in ensuring clear surgical margins [7, 8]. However, in our case, FESS provided a histological diagnosis and substantially debulked the tumour, providing significant symptomatic relief whilst preserving function. An endoscopic approach is often confined to specialised units and indicated only in lesions confined to the nasal cavity, where similar surgical margins would be expected via an external approach $[7,8]$. However, current literature regarding endoscopic management is limited and therefore its impact on overall survival and recurrence rate is promising but requires further research [7, 8]. Lymph node dissection is only performed in confirmed nodal involvement [7]. This is further supported by literature, which has previously demonstrated that nodal involvement is not a clear predictor of long-term outcome [8].

Mucosal melanoma has previously been documented to be resistant to radiotherapy, due to the recovery

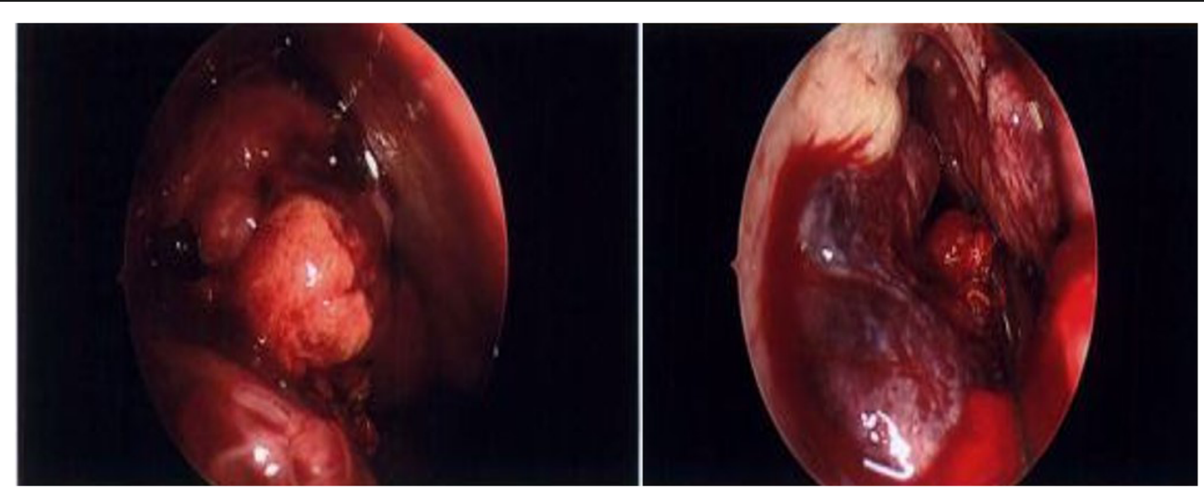

Fig. 3 Intraoperative photography during right FESS demonstrating a pigmented lesion within the right nasal cavity. The lesion underwent debulking for histological diagnosis and symptomatic relief prior to further treatment at a tertiary centre 
capability of melanoma cells post-irradiation [6]. Although adjuvant radiotherapy can be used in cases of positive surgical margins, local recurrence and palliation, there is no evidence it improves survival $[6,8]$. Intensitymodulated radiation therapy (IMRT) has however demonstrated an improvement in local disease control compared with conventional radiotherapy [6-8]. Fiveyear survival data has shown that although patients demonstrate low levels of local recurrence post-surgical management (with/without radiotherapy), clinical evidence of distant metastasis can be found in up to $80 \%$ [8]. This confirms that more research is required into metastatic disease in order to improve overall prognosis [8].

The consensus regarding the use of chemotherapy is that it is of benefit in metastatic disease as well as palliative care [6]. Literature has not shown any improvement in survival benefit when it is used in combination with surgery. Dacarbazine, paclitaxel and carboplatin have been used both individually and in combination with one another in the treatment of metastatic mucosal melanoma with overall survival benefit demonstrated [9].

The use of immunotherapy (both alone and in combination with chemotherapy) in the treatment of mucosal melanoma is novel and has shown promising results [9]. Research into immunotherapy is vital in the treatment of metastasis, especially given that there is an $80 \%$ chance over 5 years of patients developing distant disease $[9,10]$. The use of interleukin-2 and interferon-alpha have been used in the treatment of distant metastasis [7]. There has also been suggestion that neoadjuvant immunochemotherapy may reduce extent of local disease, increasing the possibility of clear margins [9]. Although current data regarding this is too limited to demonstrate clinical effectiveness, reducing tumour bulk prior to surgical treatment may reduce the risk of functional loss, as well as providing a more favourable aesthetic result.

The confirmation of genetic mutations in mucosal melanoma has demonstrated that mucosal melanoma differs genetically from its cutaneous counterpart and has subsequently led to development in monoclonal antibodies with promising results $[8,10]$. Drugs that target proto-oncogenes such as imatinib and dasatinib have been shown a significant response in overall disease control by up to $50 \%[10]$.

\section{Conclusions}

Our case highlights the importance of a thorough clinical history and examination. We recommend that any new unilateral middle ear effusion causing persistent conductive hearing loss should be treated with significant concern by clinicians. This allows patients to be diagnosed early with the best possible chance of survival. Although endoscopic approaches have been used with success in the control of local disease, no current literature found has described the use of FESS in providing symptomatic relief. Our case demonstrates that FESS is a viable surgical technique to obtain histological diagnosis whilst debulking the tumour, conferring significant symptomatic relief.

\section{Abbreviations \\ CT: Computed tomography; FESS: Functional endoscopic sinus surgery; IMRT: Intensity-modulated radiation therapy; MRI: Magnetic resonance imaging; PNS: Post nasal space; SCC: Squamous cell carcinoma}

\section{Acknowledgements}

Not applicable.

\section{Authors' contributions}

VB performed the literature review with OK and played a major part in the writing of the manuscript. OK helped obtain the relevant clinical imaging and photographs. RT provided support in the writing of the manuscript including the literature review. All authors reviewed and approved the final manuscript.

\section{Funding}

This research received no specific grant from any funding agency, commercial or not-for-profit sectors.

Availability of data and materials

Not applicable.

\section{Ethics approval and consent to participate}

Not applicable.

\section{Consent for publication}

Written informed consent was obtained from the patient for publication of this manuscript and any accompanying images.

\section{Competing interests}

The authors have no conflicts of interest to declare.

Received: 3 August 2020 Accepted: 6 November 2020

Published online: 25 November 2020

References

1. Gore MR, Zanation AM (2012) Survival in sinonasal melanoma: a metaanalysis. J Neurol Surg B Skull Base 73(suppl 3):157-162

2. Paleri V, Roland N (2016) Head and neck cancer: United Kingdom National Multidisciplinary Management Guidelines. J Laryngol Otol 130(suppl 2):1230

3. Jangard M, Hansson J, Ragnarsson-Olding B (2013) Primary sinonasal malignant melanoma: a nationwide study of the Swedish population, 19602000. Rhinology 51(suppl 1):22-30

4. Calderon-Garciduenas L, Delgado R, Calderon-Garciduenas A et al (2000) Malignant neoplasms of the nasal cavity and paranasal sinuses: a series of 256 patients in Mexico City and Monterrey. Is air pollution the missing link? Otolaryngol Head Neck Surg 122:499-508

5. Holmstrom M, Lund VJ (1991) Malignant melanomas of the nasal cavity after occupational exposure to formaldehyde. Br J Ind Med 48:9-11

6. Gilain L, Houette A, Montalban A et al (2014) Mucosal melanoma of the nasal cavity and paranasal sinuses. Eur Ann Otorhinolaryngol Head Neck dis 131:365-369

7. Clifton N, Harrison L, Bradley, P. J. et al. (2011) Malignant melanoma of nasal cavity and paranasal sinuses: report of 24 patients and literature review. J Laryngol Otol; 125(suppl 5), 479-485.

8. Ganti A, Raman A, Shay A et al (2019) Treatment modalities in sinonasal mucosal melanoma: a national cancer database analysis. Laryngoscope 00: $1-8$ 
9. Bartell HL, Bedikian AY, Papadopoulos NE et al (2008) Biochemotherapy in patients with advanced head and neck mucosal melanoma. Head Neck 30: 1592-1598

10. Crippen MM, Kilic S, Eloy JA (2018) Updates in the management of sinonasal mucosal melanoma. Curr Opin Otolaryngol Head Neck Surg 26: $52-57$

\section{Publisher's Note}

Springer Nature remains neutral with regard to jurisdictional claims in published maps and institutional affiliations.

Submit your manuscript to a SpringerOpen ${ }^{\circ}$ journal and benefit from:

- Convenient online submission

- Rigorous peer review

- Open access: articles freely available online

- High visibility within the field

- Retaining the copyright to your article

Submit your next manuscript at $\boldsymbol{\wedge}$ springeropen.com 